\title{
Do parental psychopathology and unfavorable family environment predict the persistence of social phobia?
}

\author{
Susanne Knappe ${ }^{\text {a,* }}$, Katja Beesdo ${ }^{\text {a }}$, Lydia Fehm ${ }^{\text {b }}$, Michael Höfler ${ }^{\text {a }}$, Roselind Lieb ${ }^{\text {c,d }}$, Hans- \\ Ulrich Wittchen ${ }^{\text {a,d }}$ \\ a Institute of Clinical Psychology and Psychotherapy, Technische Universitaet Dresden, Chemnitzer \\ Str. 46, 01187 Dresden, Germany \\ b Department of Psychology, Humboldt University Berlin, Germany \\ c Epidemiology and Health Psychology, University of Basel, Switzerland \\ d Max Planck Institute of Psychiatry Munich, Germany
}

\begin{abstract}
Parental psychopathology and unfavorable family environment are established risk factors for onset of offspring social phobia (SP), but their associations with the further course, e.g., persistence of the disorder, remain understudied. A community cohort of 1395 adolescents and their parents was followed-up over almost 10 years using the DIA-X/M-CIDI. Parental diagnostic interviews were supplemented by family history data. Parental rearing was retrospectively assessed by the Questionnaire of Recalled Parental Rearing Behavior in offspring, and family functioning by the Family Assessment Device in parents. Persistence measures (proportion of years affected since onset) were derived from diagnostic interviews, using age of onset, age of recency, and course information. Lack of emotional warmth and dysfunctional family functioning characteristics were associated with higher SP persistence, particularly in interaction with parental psychopathology. Predictors for SP persistence differ from those predicting SP onset. Unfavorable family environment alone and in interaction with parental disorders predict higher SP persistence.
\end{abstract}

Keywords: Social phobia, Adolescence, Parental psychopathology, Family functioning, Persistence

\section{Introduction}

Consistent with selected clinical, epidemiological, and family genetic evidence (Bosquet \& Egeland, 2006; Fyer, Mannuzza, Chapman, Liebowitz, \& Klein, 1993; McClure, Brennan, Hammen, \& Le Brocque, 2001;Merikangas, Lieb, Wittchen, \& Aveneoli, 2003; Stein, Chartier, Lizak, \& Jang, 2001), we demonstrated that parental psychopathology and negative parental rearing styles (Bruch, 1989; Gar, Hudson, \& Rapee, 2005; McClure et al., 2001; Rapee \& Spence, 2004; Woodruff-Borden, Morrow, Bourland, \& Cambron, 2002), are significantly associated with increased rates of social phobia (SP) in offspring (Lieb et al., 2000b). We recently also found first evidence that parental psychopathology, with parental SP in particular, and negative parental rearing styles independently predicted onset of SP in offspring. Interestingly, combination of these factors was associated with a higher risk for SP, as compared to the risk conferred by one of the factors alone (Knappe et al., 2009). In the current paper, we will examine whether these factors are also associated with a greater persistence of offspring SP. 
Up to date, the pathways of the development and further course after first onset of SP remain poorly understood. Retrospective and cross-sectional studies in adults have described SP as a chronic disorder with low rates of spontaneous remissions, starting in early adolescence and persisting from early adolescence into adulthood (Keller, 2003; Massion et al., 2002; Reich, Goldenberg, Vasile, Goisman, \& Keller, 1993; Wittchen \& Fehm, 2003; Yonkers, Bruce, Dyck, \& Keller, 2003). In contrast, prospective studies in children and adolescents indicate a rather oscillating course between different diagnostic levels and with low diagnostic stability on the DSMIV threshold level (Merikangas, Avenevoli, Acharyya, Zhang, \& Angst, 2002; Wittchen, Lieb, Pfister, \& Schuster, 2000), frequent transitions to subthreshold or symptomatic expressions of SP (Emmelkamp \& Wittchen, 2008), and co-occurence/incidence of other psychopathology (Beesdo et al., 2007; Essau, Conradt, \& Petermann, 2002; Last, Perrin, Hersen, \& Kazdin, 1996; Pine, Cohen, Gurley, Brook, \& Ma, 1998). Fehm, Beesdo, Jacobi, and Fiedler (2008) suggested that both threshold and subthreshold expressions of SP are associated with a considerable range of comorbid disorders and poor outcome in early adulthood. However, little is known about the factors associated with a persistent course of SP.

While parental psychopathology and unfavorable family environment are established risk factors for onset of SP, to our knowledge, no study has yet examined whether these factors also predict the further course of the disorder, namely persistence of SP symptoms after their first occurence.

Thus, we aim to study a cohort of adolescents and young adults from a representative community sample during the high risk period of SP and introduce an index to describe the persistence of SP symptoms across the high risk period of the disorder. We want to examine contribution of (1) parental psychopathology (SP and other parental disorders), (2) family environment such as parental rearing styles and family functioning, and (3) the interaction of these variables on the course, e.g., persistence of SP. Given the probable oscillating course around the diagnostic threshold of SP symptoms, and that subthreshold expressions of SP are similarly impairing and burdensome, we first consider persistence of DSMIV threshold SP, and then broaden this index also to at least subthreshold and at least symptomatic SP. This approach may also prevent from underestimating the stability of SP symptoms after initial threshold SP occurred.

\section{Methods}

\subsection{Sample}

The current study was conducted on a cohort sample of 1395 adolescents and young adults, aged 14-17 years at study entry. Data were collected as part the prospective longitudinal Early Developmental Stages of Psychopathology (EDSP) study. The EDSP study is designed as a random regional representative population sample of a German community in the metropolitan area of greater Munich to study the natural course of early stages of mental disorders and to identify risk factors for the onset and course of mental disorders. A key feature of the EDSP study is the incorporation of a special family supplement (EDSP-FS; Lieb, Isensee, Von Sydow, \& Wittchen, 2000a) to investigate familial contributions to the development of mental disorders in offspring. Detailed descriptions of the EDSP design and field procedures are reported elsewhere (Lieb et al., 2000a; Wittchen, Perkonigg, Lachner, \& Nelson, 1998). The study consists of a baseline survey conducted in 1995 (T0) with $N=3021$ individuals (response rate 71\%) of a younger (aged 14-17 years at baseline) and a older study 
cohort (18-24 years at baseline). The first follow-up (T1) (mean interval 1.64 years, S.D. = 0.19 ) was conducted only for the younger cohort $(\mathrm{N}=1228$; response rate: $87.8 \%)$, whereas the second $(\mathrm{T} 2)(\mathrm{N}=2548$, mean interval after T0 3.47 years, S.D. $=0.25$, response rate: 84.3\%) and third follow-up (T3) $(\mathrm{N}=2210$, mean interval after T0 8.38 years, S.D. $=0.65$; response rate: $73 \%$ ) was conducted for both cohorts approximately four and up to ten years after baseline. In this study, we refer to the younger cohort sample (T0: $\mathrm{N}=1395$; T1: $\mathrm{N}=$ 1228; T2: $\mathrm{N}=1169$, T3: $\mathrm{N}=1022$ ), as family environment measures were assessed only in the younger subsample. There was no selective drop out (attrition) from baseline (T0: $\mathrm{N}=$ 1395) to 10-year follow-up (T3: $\mathrm{N}=1022$ ) for SP (OR = 0.5, 95\%CI: 0.3-1.1).

Socio-demographic characteristics of the total sample are reported in Lieb et al. (2000a). In short, at baseline the majority of respondents of the younger cohort were attending school (90.1\%), only $8.5 \%$ were in job-training or employed (1.4\%). $72.7 \%$ of respondents came from a two parent household, $25.2 \%$ came from a one parent household, and the minority was living alone (1.5\%) or with others ( $0.6 \%)$. At final follow-up, $1.2 \%$ and $51.1 \%$ attended school or university, respectively, and $47.7 \%$ were employed. Also the living situations had changed, revealing that $19.7 \%$ still lived with their parents, $32.4 \%$ alone and $47.9 \%$ with others (e.g., partner, flat share).

\subsection{Diagnostic assessment}

\subsubsection{Assessment in respondents (offspring)}

Mental disorders were assessed with the computer-assisted version of the Munich-Composite International Diagnostic Interview (DIA-X/M-CIDI) (Wittchen \& Pfister, 1997), an updated version of the World Health Organization's CIDI version 1.2 (WHO, 1990, 1992). Participants were interviewed face to face by trained interviewers.

The DIA-X/M-CIDI allows for the standardized assessment of symptoms, syndromes and diagnoses of 48 mental disorders according to DSM-IV and ICD-10 criteria along with information about onset, duration, and severity. All diagnoses are based on the DSM-IV/DIA$\mathrm{X}$ algorithms. Reliability and validity are moderate to good for all the disorders covered by the DIA-X/M-CIDI. Test-retest reliability for the SP module is acceptable (kappa = .57) (Lachner et al., 1998; Reed et al., 1998).

At baseline, the DIA-X/M-CIDI was used to assess lifetime diagnoses. The follow-up surveys administered a modified version of the DIA-X/M-CIDI that covered the time interval since the last interview. To increase validity, respond lists of possible situations of social fears and a list of social fear symptoms are used (Wittchen, Kessler, \& Üstün, 2001).

The diagnostic status of respondents was categorized into four mutually exclusive groups at each assessment wave. Individuals, who denied the stem question, were classified as nonsocially anxious. Respondents who positively affirmed exclusively the DIA-X/M-CIDI stem question for ,ever having a persistent, irrational fear of, and compelling desire to avoid a situation in which the respondent attended social affairs, like going to a party or meeting,“ were labeled as symptomatic SP. Respondents were asked to give a concrete example for each item endorsed to allow for clarification. For subthreshold SP, additionally a marked and persistent fear of social or performance situations in which the person is exposed to unfamiliar people or to possible scrutiny by others (criterion A) and at least three of the following criteria had to be fulfilled: exposure to social or performance situations almost invariably provokes an immediate anxiety response (criterion B), individuals recognize that their fear is excessive or 
unreasonable (criterion C), the feared situations are avoided or else are endured with intense distress (criterion D), and social fears interfere significantly with psychosocial functioning (criterion E). As estimation of impairment due to SP may be of limited reliability in respondents of younger age, for this study, the impairment criterion (E) was only required for a SP diagnosis, when respondents were 18 years or older (Wittchen, Stein, \& Kessler, 1999). If all DSM-IV criteria (A-E) were met, a threshold SP diagnosis (DSM-IV) was assigned.

\subsubsection{Assessment in parents}

Diagnostic information on lifetime parental psychopathology was derived from both direct and indirect sources. Direct interviews were conducted with one or both parents at the first (T1) and third follow-up (T3); indirect information was collected by family history reports from their offspring at baseline, second and third follow-up (T0, T2, T3).

Parents' direct interviews were assessed using the DIA-X/M-CIDI, equal to the assessment in offspring. All interviews were conducted by trained clinical interviewers who were blind to the diagnostic findings of their offspring. Most interviews took place in the parents' homes, separate from their offspring. At T1, the primary objective according to the EDSP-FS procedures was not only to examine parental psychopathology, but also early development of the offspring, so primarily $(\mathrm{N}=1026)$ the mother was interviewed. In 27 cases, fathers were interviewed if the mother was not available (lack of time, deceased or not locatable); in three probands both mother and father were interviewed (total $\mathrm{N}=1053 / 1395$; response rate 86\%). At T3, the primary objective was to investigate first-degree relatives of offspring with a lifetime diagnosis of a depressive disorder (MDE, dysthymia), therefore only parents of these probands were invited to participate, resulting in $\mathrm{N}=130$ probands with parent interviews (122 mothers, 99 fathers, and in 91 cases both parents were interviewed directly). In the current analyses, all parents directly interviewed at either T1 or T3 (1037 mothers, 128 fathers) are included.

Indirect parental diagnoses were derived from family history items based on a modified version of the Family History Research Diagnostic Criteria (Andreasen, Endicott, Spitzer, \& Winokur, 1977). At baseline, offspring were asked M-CIDI—questions to assess the key symptoms of parental DSM-IV disorders and whether their parent sought professional help because of his or her respective symptoms. At T2 and T3, an extended version of the family history module was used, containing fully structured sections covering M-CIDI/DSM-IV criteria.

\subsubsection{Assignment of parental diagnoses}

Parental diagnoses (SP, any other anxiety disorder, depressive disorders, alcohol use disorders in either mother or father) were aggregated by using both direct (T1, T3) and indirect information (T0, T2, T3), so that for each of the 1395 respondents information about parental psychopathology was available. Direct information from T3 had highest priority and was used whenever available, followed by T1 direct information, and then followed by T3, T2 and T1 family history reports. That is, T3 family history information was only used when direct information was not given. This priority hierarchy was determined following examination of agreement patterns between family history reports and available direct interviews, which demonstrated highest agreement especially in terms of specificity at T3, compared to T2 and T1. When no indications for parental diagnoses from any of the two sources were given, parents were classified to have „no diagnosis“. 
According to their diagnosis, parents were classified into four groups: parents with SP with or without comorbid disorders (total $N=156 / 1395$; mothers: $N=143 / 1395$ (10.08\%), fathers: $N$ $=22 / 1395(1.36 \%)$, in $\mathrm{N}=9 / 1395$ (0.56\%) cases both parents were affected); parents with other anxiety disorders than SP (panic disorder, agoraphobia, generalized anxiety disorder, simple phobia, obsessive compulsive disorder, $\mathrm{N}=433 / 1395$; mothers: $\mathrm{N}=396 / 1395$ (27.70\%), fathers: $\mathrm{N}=95 / 1395$ (7.00\%), in 58/1395 (4.24\%) cases both parents were affected); parents with depressive disorders excluding SP (major depressive disorder, dysthymia, $\mathrm{N}=368 / 1395$; mothers: $\mathrm{N}=323 / 1395$ (23.12\%), fathers: $\mathrm{N}=95 / 1395$ (6.62), in 43/1395 (2.93\%) cases both parents were affected), and parents with alcohol use disorders excluding SP (alcohol abuse or dependence, $N=129 / 1395$; mothers: $N=64 / 1395$ (4.54\%), fathers: $\mathrm{N}=86 / 1395$ (6.10\%), in 14/1395 (1.12\%) cases both parents were affected). For further analyses, parental diagnostic categories were also collapsed into any parental disorder, including parental SP, any other anxiety, depressive, and alcohol use disorders ( $\mathrm{N}=$ 797/1395; mothers: $\mathrm{N}=568 / 1395$ (39.81\%), fathers: $\mathrm{N}=222 / 1395$ (15.60\%), in 114/1395 (7.96\%) cases both parents were affected).

The EDSP project and its family genetic supplement have been approved by the Ethics Committee of the Medical Faculty of the Technische Universitaet Dresden (No. EK138112002). All participants (in cases of aged 18 or younger the parents) provided written informed consent.

\subsection{Assessment of family environment}

The German Version of the Questionnaire of Recalled Parental Rearing Behavior (FEE, Schumacher, Eisemann, \& Brähler, 1999) was administered to offspring. It is based on the Swedish EMBU („Egna Minnen Beträffande Uppfostran,“ Perris, Jacobsson, Lindström, von Knorring, \& Perris, 1980), and assesses the offspring's perceived parental rearing styles with regard to parental rejection, emotional warmth, and overprotection. Reliability and validity of the FEE have been reported to be high (Arrindell et al., 1994; Schumacher et al., 1999). In the current study, chronbachs alpha was $0.69,0.75$, and 0.85 for paternal and maternal overprotection, rejection, and emotional warmth, respectively.

The McMaster Family Assessment Device (FAD, Epstein, Baldwin, \& Bishop, 1983), based on the McMaster Model of Family Functioning, was used to assess six dimensions of family functioning in directly interviewed parents: problem solving, communication, role behavior, affective responsiveness, affective involvement, and behavior control. The FAD provides scores for each of these subscales and also a ,general functioning scale, “ representing the overall family climate. In this analyses, higher scores on the FAD scales reflect dysfunctional family functioning. Reliability and validity of the FAD have been well established (Kabacoff, Miller, Bishop, Epstein, \& Keitner, 1990). In this study, chronbachs alpha ranged from 0.52 to 0.76 for the subscales, and was 0.84 for the general functioning scale.

\subsection{Persistence of social phobia}

Persistence of SP was defined as the proportion of years an individual was affected by SP symptoms given the total number of years observed or recalled after initial threshold SP occured. Using information about age of onset and age of recency of SP symptoms, a composite index was created: (1) Age of onset and age of recency information were aggregated across the assessment waves, using the lowest reported age of onset and highest reported age of recency at any of the assessment waves by convention. (2) In order to prevent from extreme recall bias leading to an overestimation of SP persistence, a more conservative 
age of onset estimation was used. When age of onset was reported before the age of 10, age of onset information was replaced by the age of 10. (3) Starting from the first report of initial threshold SP, persistence scores were calculated, irrespective of prior symptomatic or subthreshold SP conditions. Persistence scores indicate the proportion of years (\%) an individual was affected by either threshold SP, at least subthreshold, or at least symptomatic SP symptoms at subsequent assessment waves, after initial threshold SP occured. The scores range from 0 (no SP) to 1 (SP in all years observed). For example, a respondent aged 14 years at baseline (T0) participated at all subsequent assessment waves (24 years of age at T3), resulting in 10 years being observed. First onset of threshold SAD was reported at age 14. Threshold SAD was present until age 16 . From age 16 to 20 no symptoms occurred, but from age 21 to 24 criteria for subthreshold SP were met. Regarding only threshold SP, the persistence score reflects 3 years (ages 14-16) spent with threshold SP, and persistence would be $3 / 10$, indicating that $33 \%$ of the time observed threshold SP symptoms were present. This persistence rate increases to $70 \%$, when the four years (ages 21-24) of subthreshold SP were additionally considered $[(3+4) / 10]$.

\subsection{Statistical analyses}

Results (\%, means, standard deviations, coefficients) are weighted by age, gender, and geographic location at baseline to match the distribution of the original sampling frame (Lieb et al., 2000a); frequencies (Ns) are reported unweighted. The Stata Software package 10.0 (StataCorp, 2007) was used to compute robust variances, confidence intervals, and P-values (by applying the Huber-White sandwich matrix) which is required when analyses are based on weighted data (Royall, 1986). No adjustment for multiple testing was applied, because the individual tests were related to individual hypotheses and adjustment would treat them as reflecting a global hypothesis - which is questionable in substantive terms (Savitz \& Olshan, 1995).

For associations between offspring and parental psychopathology, logistic regressions provided odds ratios (ORs). Linear regression analyses were used to obtain standardized beta coefficients between parental psychopathology and family environment measures with persistence of SP in offspring, controlled for age and gender. Graphical inspections of dimensional outcomes per group did not reveal considerable departures from the assumption of a normal distribution. Scores of the FEE and FAD scales were standardized such that the standardized regression coefficient reveals the factors by which SP persistence increases for each increase of 1 standard deviation (S.D.) in the independent variable. Betas were tested for significance via T-tests (Pedhazur \& Schmelkin, 1991).

To examine interactions between parental psychopathology and family environment measures on persistence of offspring SP, parental SP or any parental disorder were entered as the first predictor into regression analyses, and the unstandardized sum sore of the respective family environment scale (FEE, FAD) were entered as the second predictor. For the interaction term as the third predictor, a product term of parental psychopathology (SP or any parental disorder) and the standardized sum score of the respective family environment scale were entered in the regression.

\section{Results}

\subsection{Presence and persistence of offspring social phobia}


Rates for DSM-IV threshold SAD were $7.48 \%$ (weighted) at any assessment wave, and $18.21 \%$ and $22.19 \%$ for subthreshold and symptomatic SP, respectively.

Respondents had a persistence index of 0.57 (S.D. $=0.29$ ) for threshold SP, and 0.64 (S.D. $=$ 0.30 ) and 0.69 (S.D. $=0.29$ ) for at least subthreshold and at least symptomatic SP, respectively.

After this brief descriptive overview, we present the findings regarding the associations between familial risk factors (parental psychopathology, family environment) and persistence of offspring SP. Analyses will first focus on persistence of threshold SP, and are then broadened to SP expressions below the diagnostic threshold, describing persistence of at least subthreshold and at least symptomatic SP in offspring.

\subsection{Does parental psychopathology predict persistence of offspring social phobia?}

For all parental disorders considered, persistence of threshold SP in offspring appeared to be higher when parents were affected by SP, any other anxiety disorder, depressive and alcohol use disorders relative to unaffected parents. Regression analyses with persistence of threshold SP as the outcome variable and the respective parental disorder as predictor were conducted to test for between-group differences. Results indicated no differences in persistence of offspring SP by parental disorders (Table 1). We also conducted the same analyses for persistence of at least subthreshold and at least symptomatic SP. Though persistence was slightly higher when also SP conditions below the DSM-IV diagnostic threshold were considered (mean range for at least subthreshold SP: 0.63-0.73; mean range for at least symptomatic SP: 0.62-0.78), no associations with parental psychopathology, e.g., no differences in persistence by parental psychopathology were found.

Though we recently found that rates of offspring threshold SP were higher in affected parents than in unaffected parents (Knappe et al., 2009), parental psychopathology did not predict persistence of the disorder in offspring.

\subsection{Does unfavorable family environment predict persistence of offspring social phobia?}

With regard to parental rearing styles (FEE, reported by offspring), no associations of parental overprotection, rejection or emotional warmth with persistence of DSM-IV threshold SP in offspring (Table 2, upper part) were found. When analyses were extended to conditions of SP below the diagnostic threshold, associations between persistence of least subthreshold (Beta = $-.25, \mathrm{~T}=-2.34, \mathrm{P}=.021$ ) and - though marginally - also persistence of at least symptomatic SP $($ Beta $=-.20, \mathrm{~T}=-1.83, \mathrm{P}=.071)$ increased with lower levels of perceived emotional warmth.

Considering family functioning (FAD, reported by parents), persistence of threshold SP was higher when family functioning was regarded as dysfunctional: FAD subscales communication (Beta $=.40, \mathrm{~T}=4.58, \mathrm{P}=.000$ ), affective overinvolvement (Beta $=.26, \mathrm{~T}=$ $2.06, \mathrm{P}=.043$ ), and general functioning (Beta $=.26, \mathrm{~T}=2.03, \mathrm{P}=.046$ ) were associated with higher persistence of offspring SP. Results were similar for persistence of at least subthreshold and at least symptomatic SP,where also affective responsiveness predicted higher persistence (subthreshold SP: Beta $=.33, \mathrm{~T}=2.62, \mathrm{P}=.001$; symptomatic SP: Beta $=$ $.26, \mathrm{~T}=2.04, \mathrm{P}=.045)$. 
As family environment measures may be influenced by parental mental health, associations with persistence of offspring SP were controlled for parental psychopathology. Results for the associations between parental rearing and persistence did not change, and lack of emotional warmth remained to be associated with persistence of subthreshold SP (Beta $=-.24, \mathrm{~T}=-2.20$, $\mathrm{P}=.030$ ). In addition, after controlling the associations between family functioning scales and persistence of offspring SP for parental psychopathology, dysfunctional communication (Beta $=.40, \mathrm{~T}=4.62, \mathrm{P}=.000)$, affective overinvolvement $($ Beta $=.33, \mathrm{~T}=2.62, \mathrm{P}=.011)$, and general functioning (Beta $=.26, \mathrm{~T}=2.07, \mathrm{P}=.042$ ) still predicted persistence of DSM-IV threshold SP symptoms. Moreover, affective responsiveness emerged as a further predictor for persistence of DSM-IV threshold SP (Beta $=.26, \mathrm{~T}=2.08, \mathrm{P}=.041)$. For persistence of at least subthreshold and at least symptomatic SP, results did not change.

\subsection{Do interactions of parental psychopathology and dysfunctional family environment contribute to social phobia persistence?}

Interested in whether the diagnostic status of either parent and negative parental rearing, and dysfunctional family functioning, respectively, interact on the persistence of offspring SP, we first report on results regarding the interaction between parental psychopathology (SP as well as other parental disorders) and rearing styles, and second, on results regarding the interaction between parental psychopathology and family functioning on persistence of offspring SP. For graphic visualization, family environment measures were dichotomized into 'low' and 'high' by median split, to indicate interactions with parental psychopathology (Figs. 1-4). Detailed tables are available upon request. Regarding offsprings' reports on parental rearing styles, an interaction was found, whereby higher levels of parental overprotection were associated with higher persistence of DSM-IV threshold SP and at least subthreshold SP in offspring, but only when SP was present in parents (DSM-IV threshold SP: Beta $=.23, \mathrm{~T}=2.06, \mathrm{P}=.043$; at least subthreshold SP: Beta $=.22, \mathrm{~T}=2.07, \mathrm{P}=.042)($ Fig. 1$)$.

For parental emotional warmth and rejection, no interactions were found. Using the broader category of 'any parental disorder' indicated that higher levels of emotional warmth were only associated with lower persistence of at least symptomatic SP in offspring in the absence of parental disorders (Beta $=.47, \mathrm{~T}=2.69, \mathrm{P}=.009$ ) (Fig. 2). In addition, an interaction with parental rejection (Beta $=-.42, \mathrm{~T}=-2.18, \mathrm{P}=.032$ ) predicted persistence of at least symptomatic SP in offspring, but not persistence of at least subthreshold or DSM-IV threshold SP (Fig. 3). Fairly the same findings occurred, when analyses were rerun for parental anxiety disorders with or without social phobia.

Parental reports on family functioning already indicated, that dysfunctional communication, affective responsiveness and affective overinvolvement were associated with higher persistence in offspring SP. Beyond these main effects, no interaction between parental SP and FAD scales on persistence of SP occured, indicating that interaction of these variables does not substantially contribute to variance of SP persistence. The association of the general functioning scale with DSM-IV threshold SP persistence was weaker and marginally significant (Beta $=.28, \mathrm{~T}=1.87, \mathrm{P}=.066$ ) after introducing the interaction term. However, using the broader category of 'any parental disorder' indicated an interaction, whereby dysfunctional problem solving was associated with higher persistence of DSM-IV threshold, at least subthreshold, and at least symptomatic SP (Beta range .91-.99), only when parental disorders were present (Fig. 4). Parental reports on dysfunctional problem solving were protective against higher persistence in the absence of parental disorders. For other family functioning scales, no interactions on persistence of offspring SP were found. Notably, also 
no interaction occurred for dysfunctional problem solving and any parental anxiety disorder with or without SP, respectively.

\section{Discussion}

\subsection{Main findings and concordance with previous studies}

We examined parental psychopathology and family environment as predictors for persistence of SP after first onset of the disorder in offspring of parents with SP and other mental disorders. The major finding is that lack of emotional warmth and dysfunctional family functioning were confirmed as independent predictors, while parental psychopathology was only associated with higher persistence of offspring SP in interaction with unfavorable environment. Notably, we examined threshold as well as subthreshold expressions of SP. On the one hand, this may have helped to increase statistical power. On the other hand, and more importantly, our approach highlights the necessity to consider SP symptoms above and below the diagnostic threshold, especially when investigating risk factors and determinants of the course of this highly prevalent anxiety disorder.

In our study, parental psychopathology was not found to have an independent contribution to increased persistence of offspring SP, while for onset, our own (Knappe et al., 2009; Lieb et al., 2000b) and other previous family (e.g., Fyer et al., 1993; McClure et al., 2001), twin (Hettema, Neale, \& Kendler, 2001; Kendler, Karkowski, \& Prescott, 1999) and high risk studies (Merikangas et al., 2003) revealed that parental mental health, and in some studies parental SP in particular, emerged as a risk factor for the onset of offspring SP. Though contradictory at first sight, it appears that parental psychopathology may play a crucial role for onset of the disorder, but is of less significance when it comes to the maintenance, e.g., persistence of symptoms. This is partly consistent with Kendler's observation from twin studies that parental psychopathology is a quite unspecific factor to promote the onset of the disorder, and its further course may be rather driven by environmental factors (also see Murray, Cooper, Creswell, Schofield, \& Sack, 2007). Yet, it is still unclear which factors predict the maintenance of anxiety disorders after their first occurence (Turner, Beidel, Roberson-Nay, \& Tervo, 2003). We found that lack of parental emotional warmth and various dimensions of family functioning, namely dysfunctional communication, affective responsiveness, and affective overinvolvement were associated with higher persistence of SP above and below the diagnostic threshold. However, studies using family functioning to predict psychopathology in offspring yielded mixed results, where some were able to distinguish between healthy and unhealthy families (Tamplin \& Goodyer, 2001), and others were not (Derisley, Libby, Clark, \& Reynolds, 2005). Tamplin and Goodyer (2001) suggested that family functioning is not necessarily a significant predictor in the etiology (e.g., onset) of the disorder, but also be relevant for its maintenance. This interpretation is supported by a recent study of our work group on the same data set (Knappe et al., 2009), where dysfunctional family functioning was not associated with lifetime incidence of SP (e.g., SP onset), whereas it predicted SP persistence in the current analyses, even after controlling for parental psychopathology.

Within these relations, it is important to consider the reciprocity of relations between parents and their offspring. Parenting behaviors potentially maintain or alter the child's anxious behavior when children's anxiety elicits parental overprotection or rejection (Burgess, Rubin, Cheah, \& Nelson, 2005; Moore, Whaley, \& Sigman, 2004; Vasey \& Dadds, 2001). But parents may also promote child anxiety by disengaging from potentially threatening situations, or by failing to encourage their children and to promote their autonomy, probably, 
and at least in part, due to their own (social anxiety) disorder (Murray et al., 2007; WoodruffBorden et al., 2002). So despite associations of family environment measures and persistence of SP in offspring were unaffected by parental psychopathology, persistence of offspring SP was particularly high when family environment measures were perceived as more negative or dysfunctional and parents were affected by psychopathology themselves. These interactions would indicate that behavior of affected parents towards their child is crucial for the further course, e.g., persistence of the disorder. Affected parents may be limited in their social resources due to their own distress, and thus have only limited capacity for assisting their offspring, e.g., in coping stressful social situations (Woodruff-Borden et al., 2002). Vice versa, parents who successfully manage their behavioral or emotional problems, would be able to provide valuable guidance and thus do not necessarily contribute to or even help to decrease persistence of SP in their offspring.

However, these interactions do not allow to disentangle the familial influence into that due to genes, shared and nonshared environment (cf. Eley \& Lau, 2005). According to findings from adoption design studies (Riggins-Caspers, Cadoret, Knutson, \& Langbehn, 2003) one would suggest that children who possess both genetic (as indicated by parental psychopathology) and psychosocial risk factors (family functioning, parental rearing styles) exhibit the highest level of SP persistence. Nevertheless, this could also be explained by gene-environment correlation, with genetic risk in the offspring leading to greater family discord and eventually parental discord (Eley \& Lau, 2005).

\subsection{Specificity of these findings for social phobia}

Extending our previous (Knappe et al., 2009) results, these findings suggest that parental mental disorders and family environment are both associated with an increased risk of onset of SP in offspring. The fact that parental SP, but not parental disorder per se interacted with negative parental rearing (overprotection) indicates at least some specificity for parental SP as a risk factor for a poorer course of SP in the offspring. In contrast, the broader category of any parental disorder showed interactions with other aspects of dysfunctional family environment (lack of emotional warmth, rejection, problem solving) on SP persistence. Taken these two findings together, they suggest that there might be two distinguishable mechanisms contributing in a complex way to patterns of onset and persistence.

Interestingly, in additional explorative analyses, similar results emerged when interactions with parental rearing were computed for any parental anxiety disorder with or without SP. From that, interactions on the course, e.g., persistence of SP findings appear to be specific for at least parental anxiety disorders. However, because of the limitations of this study (low numbers of parental SP may have limited the statistical power, use of a novel persistence measure) these findings need replication. Further, other factors that may be more specifically linked to SP persistence (e.g., clinical characteristics) were not yet covered in our present analyses. So far, our analyses were limited to persistence of SP in offspring. Future analyses will need to examine (1) whether parental psychopathology and family environment also predict persistence of other (anxiety) disorders, and (2) whether other factors such as more proximal clinical characteristics of the disorder are also associated with higher persistence.

\subsection{Implications for prevention, intervention and future studies}

Our results imply that adolescents with a family history of mental disorders and particularly those with SP, perceiving negative parental rearing styles or whose parents report dysfunctional family functioning, respectively, are at an especially high risk for persistent SP 
symptoms over many years into adulthood. These findings - if confirmed - might have important implications for targeted health care, for example considering early intervention and treatment provision in parents with SP in order to delay or even to prevent onset of SP in their offspring. Such intervention might take place as early as pre-birth of the offspring. Second, after onset of offspring SP, interventions within the family context might be of greater relevance. Family provides the (most) important context for development of the child, so families can be meaningfully involved in both the prevention and treatment of adolescent mental health problems (Robinson \& Cartwright- Hatton, 2008; Sanders \& Ralph, 2005). Parental psychopathology itself and in particular in combination with dysfunctional family functioning requires appropriate intervention, especially with regard to improvements in outcomes of their offspring (Cooper, Gallop, Willetts, \& Creswell, 2008; Manassis, 2005; Victor, Bernat, Bernstein, \& Layne, 2007). For example, Crawford and Manassis (2001) found family functioning and levels of frustration in families highly predictive of CBT outcomes in anxious children. Consideration of family issues may also have a positive effect on rates of remission and relapse (Barrett, Rapee, Dadds, \& Ryan, 1996). Though our results need replication, they support requests for the family based intervention and prevention in adolescent SP.

\subsection{Strenghts and limitations}

The major strength of this study is to examine the contribution of familial psychopathology and unfavorable family environment on the persistence of offspring SP across the high risk period of SP in a cohort of a representative community sample. However, some limitations need to be mentioned. Due to the low prevalence of SP in fathers, parent-offspringassociations may be dominated by maternal SP. Despite the prospective-longitudinal design of the EDSP study, findings disregard the temporal relationship among variables. Thus, conclusions about whether family environment characteristics precede, accompany or even follow the disorder in offspring cannot be made. Also other factors like temperament may have putative influence on parental rearing and family functioning (Burgess et al., 2005; Manassis \& Bradley, 1994) and thereby contribute to persistence of offspring SP. Offsprings' and parental reports on family environment were assessed retrospectively. However, at time of assessment, they referred to contemporary, daily experiences within the family system, as most of respondents still lived with their parents. A further critical point arises from offsprings' reports of parental rearing, that are subjective and do not allow for conclusions about actual parental rearing; similarly, parental reports on family functioning may not correspond to offsprings' views on the family climate. As parents rate the family functioning for the whole family, problems between specific dyads may not be reflected in the FAD scores. From that, our results would even underestimate the role of family environment on the persistence of SP. 


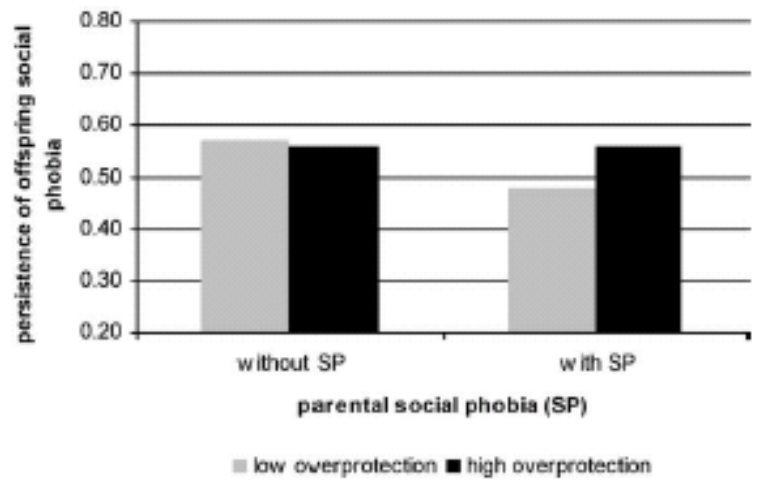

Fig 1. Interaction between parental sodal phobia and parental overprotection on persistence of offspring (at least subthreshold) socizl phobia ( $P<005$ ). Note, Higher levels of parental overprotection were assodated with higherpersistence of offspring social phobiz, especially when parents were affected by sodial phobia; in respondents without parental social phobia parental overprotection was not associated with higher persistence (bold line).

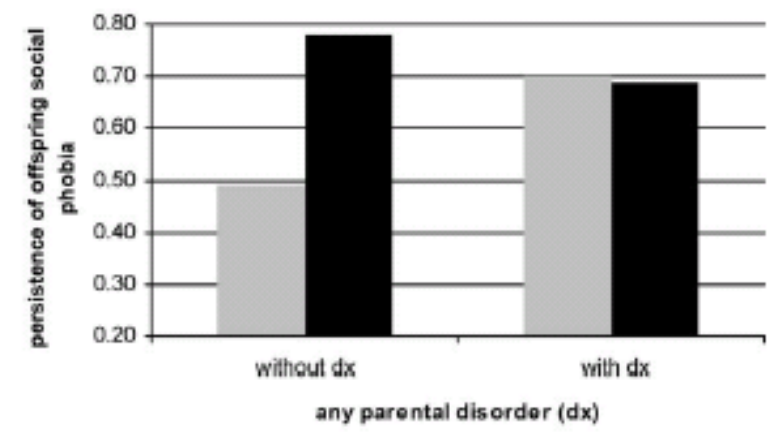

wigh emotional warmth $\mathbf{a}$ low emotional $w$ armth

Fig. 2. Interaction between any parental disorder and parental emotional warmth on persistence of offspring (at lext symptomatic) social phobia $(P<0.05$ ). Nate. Lower levels of emotional warmth were associated with higher persistence of offspring social phobia (bold line) However, higher levels of emotional warmth were only assodated with lower persistence of offspring sodal phohia if no parental disorder was present

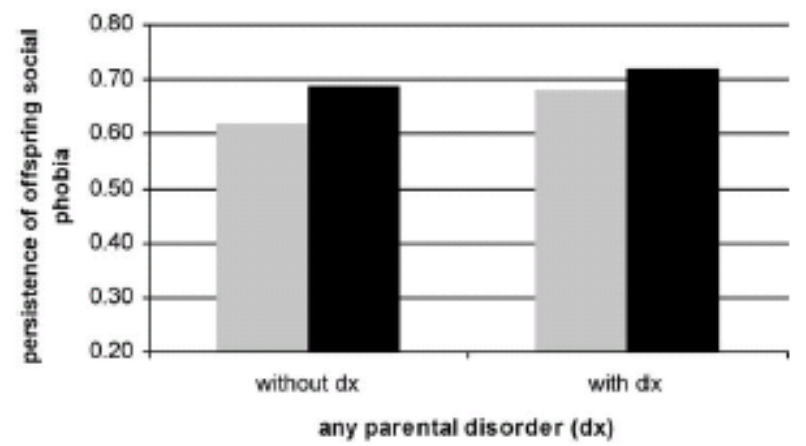

wlow rejection whigh rejection

Fig 3. Interxtion between amy parental disorder and parental rejection on persistence of offspring (at least symptomatic) social phobia $(P<005)$. Note Higher levels of parental rejection were assodiated with higher persistence of offspring social phobis. However, only in the ahsencs of any parental disorders differences in persistence by levels of parental rejection occurred. 


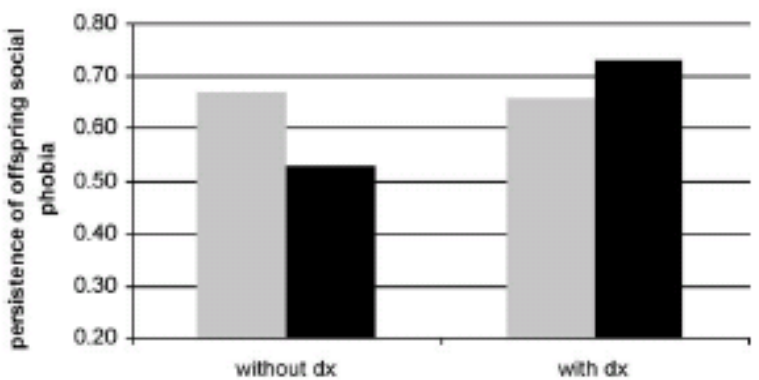

any parental disorder $(\mathrm{d} x)$

Elow problem solving $\mathbf{m}$ high problem sching

Fig 4 . Interaction between any parental disorder and parental rejection on persistence of offspring (at least symptomatic) social phobia $(P<005)$. Note. Higher (eg,dysfunctional) problemsolving was assodiated with higher persistence of off pring sodal phobia, but anly when parental disorders were present (bold line)

Table 1

Associations with parental psychopathology and persistence of offspring DSM-IV threshold sodial phobis.

\begin{tabular}{|c|c|c|c|c|c|c|c|}
\hline \multirow[t]{3}{*}{ Parental prychopathology (total $N=1395$ ) } & \multicolumn{7}{|c|}{ Persistence of offspring DSM-IV threshold social phobia } \\
\hline & \multicolumn{2}{|c|}{ Offspring of uraffected parents } & \multicolumn{2}{|c|}{ Offepring of ffected parents } & \multicolumn{3}{|c|}{ Assodiations" } \\
\hline & M & SD & M & SD. & Beta & $T$ & $\mathrm{P}$ \\
\hline Any other arociaty disorder" $(N-433)$ & 0.62 & 028 & a63 & a.28 & .04 & 039 & .01 \\
\hline Any depressive disorder" (N - 368) & 0.61 & 029 & ass & a.27 & .05 & as2 & .604 \\
\hline Any alon hal use disorder $(\mathrm{N}-129)$ & 0.63 & 028 & ast & a.28 &.$- \mathrm{B}$ & -0.29 & .770 \\
\hline
\end{tabular}

Nat: SP: social phohin. N: umveighted number; numbers do not add up as parents could also have multiple diagnoses of any other amciety, depres sive or al 6 hol use diso rider.

a Includes panic disorder, agor aphobis, gereralized aroxiety disorder, simple phobiz, obsessive compulsive disorder in either par ent

- Includes panic disorder, agor aphobia, generalized aroviety disorder,

$c$ Includes alcohol dependence or abuse in either parent.

- Beta standardized regression coefficient from linear regression, with the respective parental mental disorder as predictor, adjus ted for age and gender $P<.05$.

Table 2

Associations between parental rearing styles and family functioning and persistence of offspring DSM-N threshold social phobia

\begin{tabular}{|c|c|c|c|c|c|c|c|}
\hline \multirow[t]{2}{*}{ Family environment } & \multicolumn{7}{|c|}{ Persistence of offspring DSM-N threshold social phobia } \\
\hline & \multicolumn{2}{|c|}{$\begin{array}{l}\text { Hy low scores of family } \\
\text { enviromment" }\end{array}$} & \multicolumn{2}{|c|}{$\begin{array}{l}\text { By high scores of family } \\
\text { environment }\end{array}$} & \multicolumn{3}{|c|}{ Associations" } \\
\hline \multicolumn{8}{|l|}{ Parenting style (FEE) } \\
\hline Overprotection & as6 & $a 30$ & as6 & a.27 & .99 & 073 & .468 \\
\hline Emotional warmth & 0.48 & $a 30$ & 0.60 & 026 & -11 & -1.10 & 273 \\
\hline Rejection & 0.56 & $a \approx \theta$ & as7 & $a \geq \theta$ & .05 & aso & .618 \\
\hline Problem solving & as4 & 0.28 & a.60 & 0.27 & .13 & 092 & 359 \\
\hline Communiation & a.sz & $0 \approx 2$ & a.t3 & 0.26 & .40 & 458 & .000 \\
\hline Role behavior & a.s3 & $a \approx \theta$ & 0.59 & $a_{27}$ & .17 & 1.33 & 188 \\
\hline Affective overinvalvenent & 0.53 & 0.28 & a.62 & 0.28 & 26 & 206 & .043 \\
\hline Affective resporsiveness & a.53 & $a 27$ & 0.61 & 0.28 & 33 & 259 & .110 \\
\hline Behaviar control & a.53 & 0.30 & 060 & 0.26 & .10 & 0.87 & 309 \\
\hline Ceneral functioning & 0.52 & 0.28 & a.61 & 0.28 & 26 & 203 & .046 \\
\hline
\end{tabular}

Not: FEE: Questionnaire of Recalled Parental Rearing Behavior (Schumacher et al_,1999) and FAD: McMaster Family Assessment Device (Kahacoff et al, 1990 ) SP: sodal phobis.

A Derived by median split ( 50 th percentile) of the respective family environment scale.

"Beta standardized regression coefficient from linear regression, with the respective dimensional family environment scale as prectictor, adjusted for age and gender $P<.05$, bold prints indicate statistical significance.

\section{Acknowledgements}

This work is part of the Early Developmental Stages of Psychopathology (EDSP) Study and is funded by the German Federal Ministry of Education and Research (BMBF) project nos. 01EB9405/6, 01EB 9901/6, EB01016200, 01EB0140, and 01EB0440. Part of the field work and analyses were also additionally supported by grants of the Deutsche Forschungsgemeinschaft (DFG) LA1148/1-1, WI2246/1-1, WI 709/7-1, and WI 709/8-1. 
Principal investigators are Dr. Hans-Ulrich Wittchen and Dr. Roselind Lieb.

Core staff members of the EDSP group are Dr. Kirsten von Sydow, Dr. Gabriele Lachner, Dr. Axel Perkonigg, Dr. Peter Schuster, Dr. Michael Höfler, Dipl.-Psych. Holger Sonntag, Dipl.Psych. Tanja Brückl, Dipl.-Psych. Elzbieta Garczynski, Dr. Barbara Isensee, Dipl.-Psych. Agnes Nocon, Dr. Chris Nelson, Dipl.-Inf. Hildegard Pfister, Dr. Victoria Reed, Dipl.-Soz. Barbara Spiegel, Dr. Andrea Schreier, Dr. Ursula Wunderlich, Dr. Petra Zimmermann, Dr. Katja Beesdo, Dr. Antje Bittner, Dipl.-Psych. Silke Behrendt and Dipl.-Psych. Susanne Knappe. Scientific advisors are Dr. Jules Angst (Zurich), Dr. Jürgen Margraf (Basel), Dr. Günther Esser (Potsdam), Dr. Kathleen Merikangas (NIMH, Bethesda), Dr. Ron Kessler (Harvard, Boston) and Dr. Jim van Os (Maastricht). Appendix A. Supplementary data Supplementary data associated with this article can be found, in the online version, at doi:10.1016/j.janxdis.2009.06.010.

\section{References}

- $\quad$ Andreasen, N. C., Endicott, J., Spitzer, R. L., \& Winokur, G. (1977). The family history method using diagnostic criteria: reliability and validity. Archives of General Psychiatry, 34, 1229-1235.

- Arrindell, W. A., Perris, C., Eisemann, M., Van der Ende, J., Gaszner, P., Iwawaki, S., Maj, M., \& Zhang, J. E. (1994). Parental rearing behaviour from a cross-cultural perspective: a summary of data obtained in 14 nations. In: C. Perris, W. A. Arrindell, \& M. Eisemann (Eds.), Parenting and psychopathology (pp. 145-171). New York: Wiley \& Sons.

- Barrett, P. M., Rapee, R. M., Dadds, M. R., \& Ryan, N. D. (1996). Family enhancement of cognitive style in anxious and aggressive children. Journal of Abnormal Child Psychology, 24, 187-203.

- $\quad$ Beesdo, K., Bittner, A., Pine, D. S., Stein, M. B., Höfler, M., Lieb, R., \& Wittchen, H.-U. (2007). Incidence of social anxiety disorder and the consistent risk for secondary depression in the first three decades of life. Archives of General Psychiatry, 64, 903-912.

- Bosquet, M., \& Egeland, B. (2006). The development and maintenance of anxiety symptoms from infancy through adolescence in a longitudinal sample. Development and Psychopathology, 18, 517-550.

- Bruch, M. A. (1989). Familial and developmental antecendents of social phobia: issues and findings. Clinical Psychological Review, 9, 37-47.

- $\quad$ Burgess, K. B., Rubin, K. H., Cheah, C. S. L., \& Nelson, L. J. (2005). Behavioral inhibition, social withdrawal, and parenting. In: W. R. Crozier \& L. E. Alden (Eds.), The essential handbook of social anxiety for clinicians (pp. 99-120). New York, NY, US: John Wiley \& Sons Ltd.

- Cooper, P. J., Gallop, C., Willetts, L., \& Creswell, C. (2008). Treatment response in child anxiety is differentially related to the form of maternal anxiety disorder. Behavioural and Cognitive Psychotherapy, 36, 41-48.

- Crawford, A. M., \& Manassis, K. (2001). Familial predictors of treatment outcome in childhood anxiety disorders. Journal of the American Academy of Child \& Adolescent Psychiatry, 40, 1182-1189.

- Derisley, J., Libby, S., Clark, S., \& Reynolds, S. (2005). Mental health, coping and familyfunctioning in parents of young people with obsessive-compulsive disorder and with anxiety disorders. British Journal of Clinical Psychology, 44, 439-444.

- Eley, T. C., \& Lau, J. Y. F. (2005). Genetics and the family environment. In: J. L. Hudson \& R. M. Rapee (Eds.), Psychopathology and the family (pp. 3-19). New York, NY, US: Elsevier Science.

- Emmelkamp, P. M. G., \& Wittchen, H.-U. (2008). Stress-induced and fear circuitry disorders, specific phobias (Chapter 4). In: G. Andrews, D. Charney, P. J. Sirovatka, D. A. Regier, \& V.A.A.P.A. Arlington (Eds.), DSM V. American Psychiatric Publishing. Epstein, N. B., 
Baldwin, L. M., \& Bishop, D. S. (1983). The McMaster family assessment device. Journal of Marital and Family Therapy, 9, 171-180.

- Essau, C. A., Conradt, J., \& Petermann, F. (2002). Course and outcome of anxiety disorders in adolescents. Journal of Anxiety Disorders, 16, 67-81.

- Fehm, L., Beesdo, K., Jacobi, F., \& Fiedler, A. (2008). Social phobia above and below the diagnostic threshold: prevalence, comorbidity and impairment in the general population. Social Psychiatry \& Psychiatric Epidemiology, 43, 257-265.

- $\quad$ Fyer, A. J., Mannuzza, S., Chapman, T. F., Liebowitz, M. R., \& Klein, D. F. (1993). A direct interview family study of social phobia. Archives of General Psychiatry, 50, 286-293.

- Gar, N. S., Hudson, J. L., \& Rapee, R. M. (2005). Family factors and the development of anxiety disorders. In: J. L. Hudson \& R. M. Rapee (Eds.), Psychopathology and the family (pp. 125-145). New York, NY, US: Elsevier Science.

- Hettema, J. M., Neale, M. C., \& Kendler, K. S. (2001). A review and meta-analysis of the genetic epidemiology of anxiety disorders. American Journal of Psychiatry, 158,1568-1578.

- Kabacoff, R. I., Miller, I. W., Bishop, D. S., Epstein, N. B., \& Keitner, G. I. (1990). A psychometric study of the McMaster family assessment device in psychiatric, medical, and non-clinical samples. Journal of Family Psychology, 3, 341-439.

- Keller, M. B. (2003). The lifelong course of social anxiety disorder: a clinical perspective. Acta Psychiatrica Scandinavica, 108(Suppl. 417), 85-94.

- Kendler, K. S., Karkowski, L. M., \& Prescott, C. A. (1999). Fears and phobias: reliability and heritability. Psychogical Medicine, 29, 539-553.

- Knappe, S., Lieb, R., Beesdo, K., Fehm, L., Low, N. C. P., Gloster, A. T., \& Wittchen, H.-U. (2009). The role of parental psychopathology and family environment for social phobia in the first three decades of life. Depression and Anxiety, 26(4), 363-370.

- $\quad$ Lachner, G., Wittchen, H. U., Perkonigg, A., Holly, A., Schuster, P., Wunderlich, U., Turk, D., Garczynski, E., \& Pfister, H. (1998). Structure, content and reliability of the Munich Composite International Diagnostic Interview (M-CIDI) substance use sections. European Addiction Research, 4, 28-41.

- $\quad$ Last, C. G., Perrin, S., Hersen, M., \& Kazdin, A. E. (1996). A prospective study of childhood anxiety disorders. Journal of the American Academy of Child and Adolescent Psychiatry, 35, 1502-1510.

- $\quad$ Lieb, R., Isensee, B., Von Sydow, K., \& Wittchen, H.-U. (2000a). EDSP—an methodological update. European Addiction Research, 6, 170-182.

- $\quad$ Lieb, R., Wittchen, H. U., Höfler, M., Fuetsch, M., Stein, M. B., \& Merikangas, K. R. (2000b). Parental psychopathology, parenting styles and the risk of social phobia in offspring: a prospective-longitudinal community study. Archives of General Psychiatry, 57, 859-866.

- Manassis, K. (2005). Family involvement in psychotherapy: what’s the evidence. In: J. L. Hudson \& R. M. Rapee (Eds.), Psychopathology and the family (pp. 283-300). New York, NY, US: Elsevier Science.

- Manassis, K., \& Bradley, S. J. (1994). The development of childhood anxiety disorders: toward an integrated model. Journal of Applied Developmental Psychology, 15, 345-366.

- Massion, A. O., Dyck, I. R., Shea, M. T., Phillips, K. A., Warshaw, M. G., \& Keller, M. B. (2002). Personality disorders and time to remission in generalized anxiety disorder, social phobia, and panic disorder. Archives of General Psychiatry, 59, 434-440.

- McClure, E. B., Brennan, P. A., Hammen, C., \& Le Brocque, R. M. (2001). Parental anxiety disorders, child anxiety disorders, and the perceived parent-child relationship in an Australian high risk sample. Journal of Abnormal Child Psychology, 29, 1-10.

- $\quad$ Merikangas, K. R., Avenevoli, S., Acharyya, S., Zhang, H., \& Angst, J. (2002). The spectrum of social phobia in the Zurich cohort study of young adults. Biological Psychiatry, 51,81-91.

- Merikangas, K. R., Lieb, R., Wittchen, H.-U., \& Aveneoli, S. (2003). Family and high-risk studies of social anxiety disorder. Acta Psychiatrica Scandinavica, 108(Suppl. 147), 28-37.

- Moore, P. S., Whaley, S. E., \& Sigman, M. (2004). Interactions between mothers and children: impacts of maternal and child anxiety. Journal of Abnormal Psychology, 113, 471476. 
- Murray, L., Cooper, P., Creswell, C., Schofield, E., \& Sack, C. (2007). The effects of maternal social phobia on mother-infant interactions and infant social responsiveness. Journal of Child Psychology and Psychiatry, 48, 45-52.

- Pedhazur, E. J., \& Schmelkin, L. P. (1991). Measurement, design, and analysis: an integrated approach. Hillsdale, NJ, England: Lawrence Earlbaum Associates Inc.

- Perris, C., Jacobsson, L., Lindström, H., von Knorring, L., \& Perris, H. (1980). Development of a new inventory for assessing memories of parental rearing behavior. Acta Psychiatrica Scandinavica, 61, 265-274.

- Pine, D. S., Cohen, P., Gurley, D., Brook, J., \& Ma, Y. (1998). The risk for early-adulthood anxiety and depressive disorders in adolescents with anxiety and depressive disorders. Archives of General Psychiatry, 55, 56-64.

- Rapee, R. M., \& Spence, S. H. (2004). The etiology of social phobia: empirical evidence and an initial model. Clinical Psychological Review, 24, 737-767.

- $\quad$ Reed, V., Gander, F., Pfister, H., Steiger, A., Sonntag, H., Trenkwalder, C., Sonntag, A., Hundt, W., \& Wittchen, H.-U. (1998). To what degree the Composite International Diagnostic Interview (CIDI) correctly identifies DSM-IV disorders? Testing validity issues in a clinical sample. International Journal of Methods in Psychiatric Research, 7, 142-155.

- $\quad$ Reich, J., Goldenberg, I., Vasile, R., Goisman, R., \& Keller, M. (1993). A prospective followalong study of the course of social phobia. Psychiatry Research, 54, 249-258.

- $\quad$ Riggins-Caspers, K. M., Cadoret, R. J., Knutson, J. F., \& Langbehn, D. (2003). Biologyenvironment interaction and evocative biology-environment correlation: contributions of harsh discipline and parental psychopathology to problem adolescent behaviors. Behavior Genetics, 33, 205-220.

- $\quad$ Robinson, R., \& Cartwright-Hatton, S. (2008). Maternal disciplinary style with preschool children: associations with children's and mother's trait anxiety. Behavioural and Cognitive Psychotherapy, 36, 49-59.

- Royall, M. (1986). Model robust confidence intervals using maximum likelihood estimators. International Statistical Review, 54, 221-226.

- Sanders, M. R., \& Ralph, A. (2005). Family intervention and prevention of behavioral and emotional disorders in childhood and adolescence. In: J. L. Hudson \& R. M. Rapee (Eds.), Psychopathology and the family (pp. 323-350). New York, NY, US: Elsevier Science.

- Savitz, D. A., \& Olshan, A. F. (1995). Multiple comparisons and related issues in the interpretation of epidemiologic data. American Journal of Epidemiology, 142,904-908.

- Schumacher, J., Eisemann, M., \& Brähler, E. (1999). Rückblick auf die Eltern: der Fragebogen zum erinnerten elterlichen Erziehungsverhalten [Looking back on parents: the questionnaire of recalled parental rearing behavior]. Diagnostica, 45, 194-204.

- StataCorp. (2007). Stata Statistical Software: Release 10. College Statio, TX: StataCorp. Stein, M. B., Chartier, M. J., Lizak, M. V., \& Jang, K. L. (2001). Familial aggregation of anxiety-related quantitative traits in generalized social phobia. American Journal of Medical Genetics, 105, 79-83.

- Tamplin, A., \& Goodyer, I. M. (2001). Family functioning in adolescents at high and low risk for major depressive disorder.. European Child \& Adolescent Psychiatry, 10(3),170-179.

- $\quad$ Turner, S. M., Beidel, D. C., Roberson-Nay, R., \& Tervo, K. (2003). Parenting behaviors in parents with anxiety disorders. Behaviour Research and Therapy, 41, 541-554.

- Vasey, M. W., \& Dadds, M. R. (2001). An introduction to the developmental psychopathology of anxiety. In: M. W. Vasey \& M. R. Dadds (Eds.), The developmental psychopathology of anxiety (pp. 3-26). New York: Oxford University Press.

- Victor, A. M., Bernat, D. H., Bernstein, G. A., \& Layne, A. E. (2007). Effects of parent and family characteristics on treatment outcome of anxious children. Journal of Anxiety Disorders, 21, 835-848.

- Wittchen, H.-U., \& Fehm, L. (2003). Epidemiology and natural course of social fears and social phobia. Acta Psychiatrica Scandinavica, 108, 4-18.

- $\quad$ Wittchen, H.-U., Kessler, R. C., \& Üstün, T. B. (2001). Properties of the Composite International Diagnostic Interview (CIDI) for measuring mental health outcome. In: M. 
Tansella \& G. Thornicroft (Eds.), Mental health outcome measures (pp. 212-227). London: Gaskell.

- Wittchen, H.-U., Lieb, R., Pfister, H., \& Schuster, P. (2000). The waxing and waning of mental disorders: evaluating the stability of syndromes of mental disorders in the population. Comprehensive Psychiatry, 41(Suppl. 1), 122-132.

- Wittchen, H.-U., Stein, M. B., \& Kessler, R. C. (1999). Social fears and social phobia in a community sample of adolescents and young adults: prevalence, risk factors, and comorbidity. Psychological Medicine, 29, 309-323.

- Wittchen, H.-U., Perkonigg, A., Lachner, G., \& Nelson, C. B. (1998). Early Developmental Stages of Psychopathology Study (EDSP): objectives and design. European Addiction Research, 4, 18-27.

- Wittchen, H.-U., \& Pfister, H. (1997). DIA-X-Interviews: manual für Screening Verfahren und Interview; Interviewheft Längsschnittuntersuchung (DIA-X-Lifetime); Ergänzungsheft (DIA-X-Lifetime); Interviewheft Querschnittsuntersuchung (DIA-X-12 Monate); Ergänzungsheft (DIA-X-12 Monate); PC-Programm zur Durchführung des Interviews (Längsund Querschnittsuntersuchung); Auswertungsprogramm. Frankfurt: Swets \& Zeitlinger.

- Woodruff-Borden, J., Morrow, C., Bourland, S., \& Cambron, S. (2002). The behavior of anxious parents: examining mechanisms of transmission of anxiety from parent to child. Journal of Clinical Child and Adolescent Psychology, 31, 364-374.

- Yonkers, K. A., Bruce, S. E., Dyck, I. R., \& Keller, M. B. (2003). Chronicity, relapse, and illness - course of manic disorder, social phobia, and generalized anxiety disorder: findings in men and women from 8 years of follow-up. Depression and Anxiety, 17,173-179. 